\title{
Progesterone Provides the Pleiotropic Neuroprotective Effect on Traumatic Brain Injury Through the Nrf2/ARE Signaling Pathway
}

\author{
Mei Zhang ${ }^{1} \cdot$ Jianyue Wu $^{1} \cdot$ Haojun Ding ${ }^{1} \cdot$ Wentian $\mathrm{Wu}^{1} \cdot$ Guomin Xiao $^{1}$
}

Published online: 19 December 2016

(C) The Author(s) 2016. This article is published with open access at Springerlink.com

\begin{abstract}
Objective This study was to investigate the role of Nrf2/ ARE signaling pathway in the pleiotropic neuroprotective effect of progesterone (PROG) on traumatic brain injury (TBI).

Methods The Nrf2-knockout (Nrf2-/-) and C57 mice were respectively subjected to a lateral cortical impact injury caused by a free-falling object and randomly divided into three groups: sham-operated, trauma, and trauma + PROG treatment group. The PROG treatment group was given PROG $(32 \mathrm{mg} / \mathrm{kg}$ of body weight, intraperitoneal injection) immediately after injury. For all groups, a series of brain samples were obtained after trauma at 24 and $72 \mathrm{~h}$, respectively. The cerebral edema was evaluated; the expression of IL-1 $\beta$, IL- 6 , and TNF- $\alpha$ was measured using ELISA array, and the apoptosis index was detected by TUNEL. Flow cytometry was used to detect the intracellular calcium concentration.

Results The water content, the apoptosis index, the levels of inflammatory cytokine, and the intracellular calcium ion were significantly decreased with the PROG treatment in C57 mice with TBI model. However, the effect of PROG on TBI was not found in the Nrf2-/- mouse model of TBI.

Conclusions PROG reduced cerebral edema, apoptosis, inflammatory reaction, and intracellular calcium ion overload effects after TBI. These beneficial effects were not seen in the Nrf2-/- mouse model of TBI. The results from this study suggested that the Nrf2/ARE signal pathway may
\end{abstract}

Guomin Xiao

xiao_gm@126.com

1 Department of Neurosurgery, Affiliated Hospital of Hangzhou Normal University, Hangzhou 310015, China be involved in the pleiotropic neuroprotective effect of PROG on TBI.

Keywords Progesterone - Nrf2/ARE signaling pathway · Traumatic brain injury $\cdot$ Neuroprotection .

Nrf2 gene knockout · In vivo

Traumatic brain injury (TBI) is a leading cause of morbidity and mortality in young adults, resulting in large direct and indirect social loss [1]. TBI is caused by both primary injury due to the initial biomechanical effects force on brain tissues and subsequent secondary injury due to activation of several pathophysiologic cascades. Brain damage and dysfunction after TBI have been attributed to a large extent to the secondary neurological injury mechanisms. It is recognized that the pathophysiology of secondary brain injury is a complex cascade of molecular and biochemical events after the onset of insult, such as neuronal excitotoxicity [2, 3], intracellular $\mathrm{Ca}^{2+}$ influx [4], free radicals, and lipid peroxidation [5, 6], exacerbated inflammatory response $[7,8]$, or subsequent neuronal cell death via necrosis and apoptosis $[9,10]$.

Research has shown that progesterone (PROG) has substantial pleiotropic properties as a neuroprotective agent in both animal models and humans [11, 12]. PROG attenuates glutamate excitotoxicity [13], modulates apoptotic pathways [14], reduces membrane lipid peroxidation [15], and limits inflammation after TBI [16]. PROG is synthesized by oligodendrocytes and some neurons in the brains of both sexes $[17,18]$. PROG receptor is widely expressed in the central nervous system, so various brain regions are the normal targets of PROG [19]. PROG can also elicit its neuroprotective effects via non-genomic mechanisms such as through the activation of signal 
transduction pathways. Those second signal transduction systems are known to be activated by PROG include cAMP/PKA [20], MAPK (ERK1/2) [21, 22], TLRs/NF-кB [23], and the PI-3K/Akt signaling pathway [17]. Activation of such signaling pathways indicates its neuroprotective effects on brain injury. However, the various pathways that mediate simultaneously the pleiotropic neuroprotective effect of PROG on TBI are still unclear.

Nuclear factor erythroid-2p45-related factor 2 (Nrf2) is the family of transcription factors and binds antioxidant response element (ARE) in the promoter regions of antioxidant genes and phase II detoxification enzymes, which has been reported to be a pleiotropic regulator in the cellular defense mechanisms [24, 25]. Under basal conditions, Nrf2 is localized within the cytoplasm by binding to the cytosolic regulatory protein Keap1. Following activation, Nrf2 translocates to the nucleus where it binds to ARE, and enables gene transcription [26].

Despite the demonstrated role of PROG in neuroprotection on TBI, none of the previous studies focused on the Nrf2/ARE signaling pathway in relation to TBI. We hypothesized that the effect of PROG on activating the Nrf2/ARE signaling pathway was the mechanism whereby PROG provides the pleiotropic neuroprotective effect on TBI. The aim of this study was to investigate whether PROG activates Nrf2/ARE signaling pathway in a mouse model of TBI.

\section{Methods}

\section{Animals}

A total of 36 C57 mice (obtained from Hangzhou Normal University, Hangzhou, China), weighing $25 \pm 2 \mathrm{~g}$, were housed four to a cage in air-filtered, temperature-controlled units with 12-h light/dark cycle and regular food and water supply. Breeding pairs of 36 Nrf2--deficient ICR mice were obtained from Nanjing Qingzhilan Techonolgy Co., Ltd (Nanjing, China). Homozygous wild-type Nrf2+/+ mice and $\mathrm{Nrf} 2-/$--deficient mice were generated from inbred heterozygous Nrf2 $+/-$ mice [27]. Genotypes of Nrf2-/ - and Nrf2 $+/+$ mice were confirmed by polymerase chain reaction amplification of genomic DNA isolated from the blood. PCR amplification was performed using three different primers, 5'-TGGACGGGACTATTGAAGGCTG-3' (sense for both genotypes), 5'CGCCTTTTCAGTAGATGGAGG-3' (antisense for wildtype), and 5'-GCGGATTGACCGTAATGGGATAGG-3' (antisense for LacZ). The study protocol was approved by the Committee of Animal Care and Use for Research and Education (CACURE) of the Affiliated Hospital of Hangzhou Normal University and complied with the National
Institutes of Health (NIH) guidelines for the care and use of laboratory animals.

\section{Models of Cortical Contusion Trauma}

All mice were anesthetized with pentobarbital sodium $(60 \mathrm{mg} / \mathrm{kg})$ through intraperitoneal injection and fixed on a stereotaxic instrument. The skin of the right hemisphere was opened to expose the skull, a hole was drilled into the skull at the level of the parietal cortex $(2 \mathrm{~mm}$ to the lambda, $2 \mathrm{~mm}$ to the fontanel, and $2 \mathrm{~mm}$ to the sagittal suture), and the exposed dura was kept intact. Trauma was induced by a modification of the Feeney's weight-drop model [28] in which a free-falling weight drops onto the exposed intact cranial dura to produce a standardized parietal contusion (weighing $20 \mathrm{~g}$, falling from $12 \mathrm{~cm}$ height). After trauma, the skull hole is closed with bone wax and the scalp was sutured. The sham-operated mice underwent the same surgical procedure without being exposed to percussion injury.

\section{Experimental Protocol}

The mice were randomly assigned to three groups: (1) sham-operated group (sham); (2) trauma group (TBI); and (3) trauma + PROG treatment group (TBI + PROG). PROG (purchased from Xianju Pharmaceutical Co., Ltd, China) dissolved in $50 \%$ dimethyl sulphoxide $(10 \mathrm{mg} / \mathrm{ml})$ and $50 \%$ saline was immediately given as an intraperitoneal injection in TBI + PROG group after trauma ( $32 \mathrm{mg} / \mathrm{kg}$ body weight). Both sham and TBI groups received the same volume/weight of vehicle injection only. The mice were killed by decapitation at 24 and $72 \mathrm{~h}$, respectively, after injury.

\section{Brain Tissue Water Content Measurement}

A part of the mice brain (about $5 \mathrm{~mm} \times 5 \mathrm{~mm} \times 5 \mathrm{~mm}$ tissue cubes) was excised from the parietal cortex directly adjacent to the lesion core. The wet weight (WW) was rapidly measured with a chemical balance. The tissue was then dried in a desiccator oven at $100{ }^{\circ} \mathrm{C}$ for $24 \mathrm{~h}$ to reach constant dry weight (DW). The tissue water content was calculated as $[(\mathrm{WW}-\mathrm{DW}) / \mathrm{WW}] \times 100 \%$.

\section{TUNEL Labeling}

The sample cortex surrounding the contusion area or surrounding the parietal craniotomy in the sham group was collected. Formalin-fixed tissue was embedded in paraffin and sectioned at $4 \mu \mathrm{m}$ with a microtome. The paraffinembedded sections were also detected for apoptotic cells by terminal deoxynucleotidyl transferase dUTP nick-end- 
labeling (TUNEL) staining. The procedures were performed according to the instructions provided with the kit (Nanjing KaiJi Technology Development Co., Ltd). Paraffin sections of $5 \mu \mathrm{m}$ thick were deparaffinized, and endogenous peroxidase was blocked by immersing the sections in phosphate-buffered saline (PBS) containing $0.5 \% \mathrm{H}_{2} \mathrm{O}_{2}$ for $15 \mathrm{~min}$. For proteins nuclei stripping, tissue sections were incubated with proteinase $\mathrm{K}(20 \mu \mathrm{g} /$ $\mathrm{ml}$ ) for $10 \mathrm{~min}$. The sections were incubated with a mixture of terminal deoxynucleotidyl transferase (TdT) and reaction buffer containing digoxigenin-labeled dUTP. Hematoxylin staining was performed followed by incubation with anti-digoxigenin antibody conjugated with peroxidase. Positive cells containing the fragmented nuclear chromatin which is a characteristic of apoptosis have brown-stained nuclei. The extent of brain damage was evaluated by the apoptotic index (AI), which was the average number of TUNEL-positive cells in each section counted in 10 microscopic fields (at $400 \times$ magnification).

\section{Multiplex Cytokine Enzyme-Linked Immunosorbent Assay}

A 3-mm coronal section was taken from the injured area over the parietal cortex, accurately weighed, snap-frozen in liquid nitrogen, and stored at $-70{ }^{\circ} \mathrm{C}$. Frozen brain samples were homogenized in $1 \mathrm{~mL}$ of buffer containing $1 \mathrm{mmol} / \mathrm{L}$ of PMSF, $1 \mathrm{mg} / \mathrm{L}$ of pepstatin $\mathrm{A}, 1 \mathrm{mg} / \mathrm{L}$ of aprotinin, and $1 \mathrm{mg} / \mathrm{L}$ of leupeptin in PBS solution $(\mathrm{pH}$ 7.2) with a glass homogenizer and then centrifuged at $12,000 \mathrm{~g}$ for $20 \mathrm{~min}$ at $4{ }^{\circ} \mathrm{C}$. The supernatant was then collected, and total protein was determined by the Bradford method. The levels of inflammatory cytokines were quantified using enzyme-linked immunosorbent assay (ELISA) kits specific for rats according to the manufacturers' instructions (Uscnk, USA). The cytokine contents in the brain samples were expressed as picograms of antigen per milligram of protein.

\section{Measurement of Intracellular Free Calcium $\left(\left[\mathrm{Ca}^{2+}\right]_{i}\right)$ by Flow Cytometry}

The brain samples were washed twice with PBS, detached with a $0.125 \%$ trypsin treatment $\left(37^{\circ} \mathrm{C}, 15 \mathrm{~min}\right)$, and further washed three times with PBS. For $\left[\mathrm{Ca}^{2+}\right]_{i}$ detection, cell suspensions were incubated with fura-4acetoxymethyl ester (final concentration $5 \mu \mathrm{mol} / \mathrm{L}$, Gibco, USA) in the darkness at $37^{\circ} \mathrm{C}$ for $40 \mathrm{~min}$. Then cell suspensions were centrifuged at $1500 \mathrm{r} / \mathrm{min}$ for $5 \mathrm{~min}$. After washing once in $2 \mathrm{~mL}$ PBS, the cells were resuspended in $0.5 \mathrm{~mL}$ PBS. The fluorescence of 10000 cells was analyzed by flow cytometry $(488 \mathrm{~nm})$. The relative fluorescence intensity of Fluo-1 was used as the indication of $\left[\mathrm{Ca}^{2+}\right]_{i}$ quantity.

\section{Statistical Analysis}

All data were presented as mean \pm standard deviation. SPSS 12.0 (SPSS Inc., Chicago, IL) was used for statistical analysis of the data. For evaluation data, repeated-measures analyses of variance (two-way or one-way as appropriate) and $t$ tests were utilized to determine statistical differences. A Holm-Sidak method for multiple comparisons post hoc test was used to determine data points with significant differences. Statistical significance was inferred at $P<0.05$.

\section{Results}

\section{Brain Tissue Water Content Measurement}

As shown in Fig. 1, in C57 mice with acute brain injury model, brain tissue water content adjacent to the lesion site in the injured group was significantly increased when compared to the sham group at 24 and $72 \mathrm{~h}$ after trauma, while tissue water content was not increased in PROG treatment group when compared with the sham group at each time points after trauma. Tissue water content in the TBI + PROG group was significantly reduced compared to the injured group at the same time after trauma. However, in the Nrf2-I- mouse model of brain injury, there was no statistical significance between the TBI + PROG treatment group and the trauma group with the water content of brain at 24 and $72 \mathrm{~h}$ after injury. PROG postinjury administration did not attenuate cerebral edema after trauma in the Nrf2-/- mouse model of TBI.

\section{Apoptotic Index Measured by TUNEL Labeling}

The number of apoptotic profiles was measured by TUNEL staining. Similar to the brain tissue water content measurement, PROG post-injury administration did not reduce nerve cell death after trauma in the Nrf2-/- mouse model of TBI. As shown in Fig. 2, in the C57 mouse model of TBI, few TUNEL-positive apoptotic cells were found in brains of the sham group. The apoptotic index in the cortex surrounding the injured site was found to be significantly increased compared to the sham group animals at 24 and $72 \mathrm{~h}$ after trauma in the TBI group. In TBI + PROG group, when compared to the TBI group, the apoptotic index in the cortex surrounding the injured site was significantly decreased. However, in the Nrf2-/- mouse model of TBI, there was no statistical significance between the TBI + PROG treatment group and the TBI group with 
$\mathbf{C 5 7}$

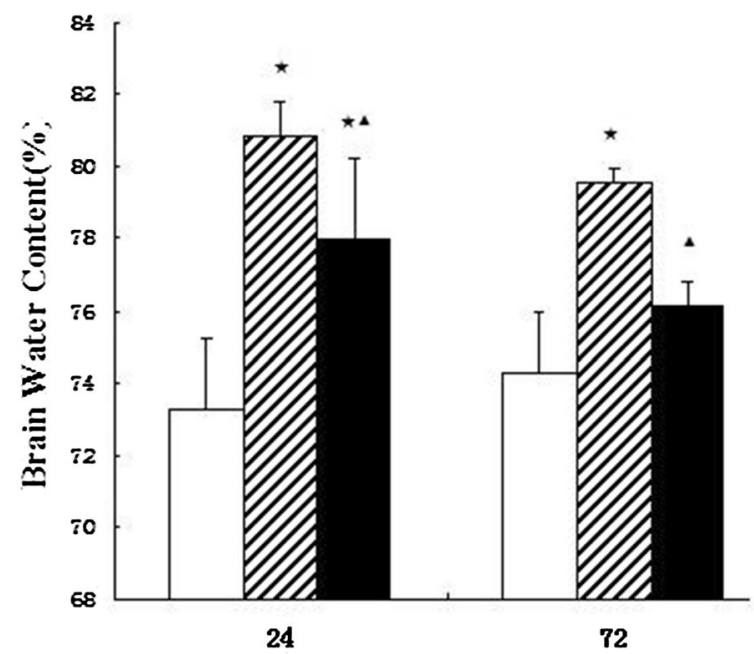

Nrf2-/-

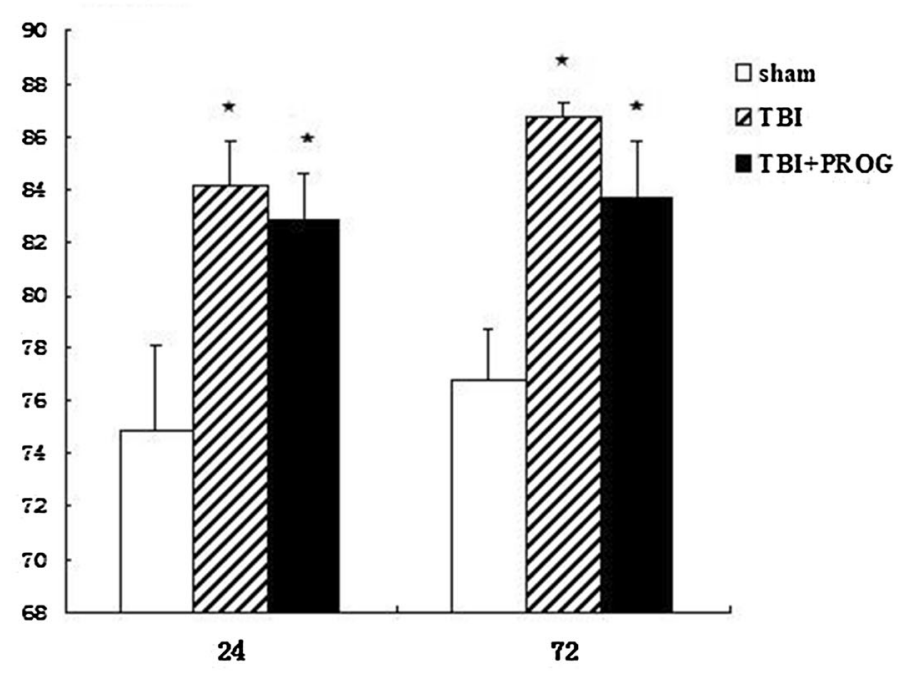

Time following trauma(h)

Fig. 1 Alterations in brain tissue water content adjacent to the lesion site in sham group, TBI group, and TBI + PROG group $(n=12)$ between the C57 mice and Nrf2-/- mouse model of brain injury at 24 and $72 \mathrm{~h}$ after injury. The right-hand side illustrates the Nrf2-I-

the apoptotic index in the cortex surrounding the injured site at 24 and $72 \mathrm{~h}$ after injury. These results indicated that PROG administration following TBI leads to less cell death in $\mathrm{Nrf} 2+/+$ mice but not in mice lacking the Nrf2 gene.

\section{Multiplex Cytokine levels Quantified with ELISA Array}

Levels of cytokines IL-1 $\beta$, IL-6, and TNF- $\alpha$ in brain tissue lysate at 24 and $72 \mathrm{~h}$ after injury measured using a commercially available ELISA array are shown in Fig. 3. In C57 mice with TBI model, the inflammatory cytokine(IL-1 $\beta$, IL-6, and TNF- $\alpha$ ) levels in sham mice brain tissue at each time point after injury were consistently presented in a low background. All three measured cytokine levels exhibited significant increases in different time points. PROG treatment did produce significant reductions in the injury-induced up-regulation of IL-1 $\beta$, IL-6, and TNF- $\alpha$ expression. However, in the Nrf2-/mouse model of TBI, PROG treatment did not reduce IL-1 $\beta$, IL-6, and TNF- $\alpha$ expression in brain tissues at 24 and $72 \mathrm{~h}$ after injury compared to the trauma group animals.

\section{$\left[\mathrm{Ca}^{2+}\right]_{i}$ Measured by Flow Cytometry}

As shown in Fig. 4, the flow cytometric analysis showed a marked increase of the relative fluorescence intensity of mouse model of TBI. The left-hand side is the C57 mouse model of TBI. The brain tissue water content is given as the mean \pm SE. ${ }^{*} P<0.01$ versus sham control and $\boldsymbol{\Delta}_{P}<0.05$ versus TBI vehicletreated injured mice

$\left[\mathrm{Ca}^{2+}\right]_{i}$ in the TBI group of $\mathrm{C} 57$ mice brain tissues at 24 and $72 \mathrm{~h}$ after injury. Compared to the TBI group, the relative fluorescence intensity of $\left[\mathrm{Ca}^{2+}\right]_{i}$ in the TBI + PROG group at the same time points after injury was significantly reduced. However, in the Nrf2-l- mouse model of TBI, there was no statistical significance between the TBI + PROG treatment group and the trauma group with the relative fluorescence intensity of $\left[\mathrm{Ca}^{2+}\right]_{i}$ in brain at 24 and $72 \mathrm{~h}$ after injury. Blockade of TBI-induced $\left[\mathrm{Ca}^{2+}\right]_{i}$ by PROG in the C57 mice with TBI model was not observed in the Nrf2-/- mouse model of brain injury.

\section{Discussion}

This study examined the role of Nrf2/ARE signaling pathway in the pleiotropic neuroprotective effect of PROG on TBI. We found that PROG has neuroprotective properties in C57 mice with acute brain injury model. Clearly, the mechanism of PROG in neuroprotection does not target a single aspect of the secondary injury cascade following TBI, but it instead works through multiple mechanisms to enhance neural repair caused by head injury; as such, PROG reduced cerebral edema, apoptosis, inflammatory reaction, and intracellular calcium ion overload effects after TBI. However, in the Nrf2-knockout mice model, our results showed that the loss of Nrf2 in vivo lessens the 
C57

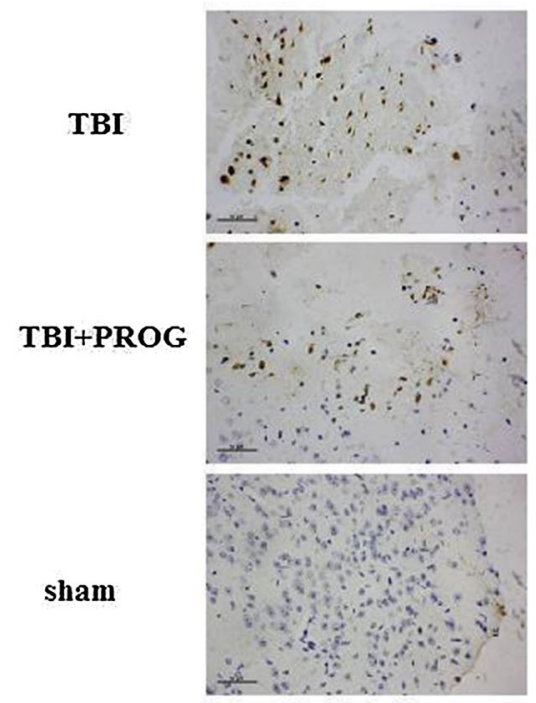

24
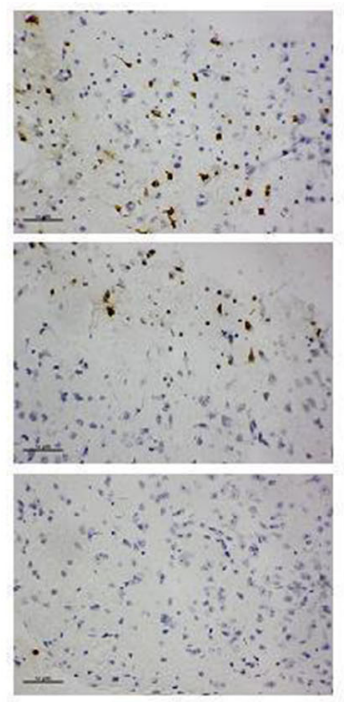

72
Nrf2-/
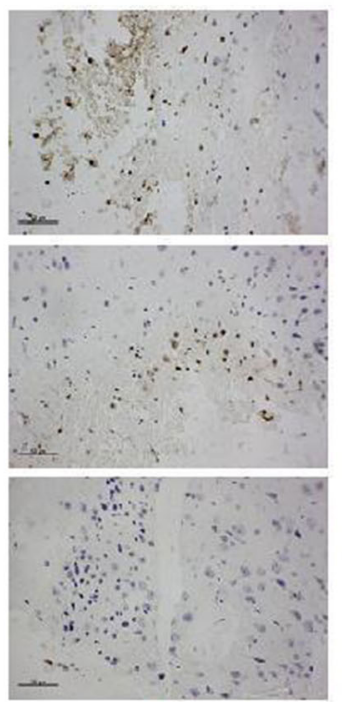

24
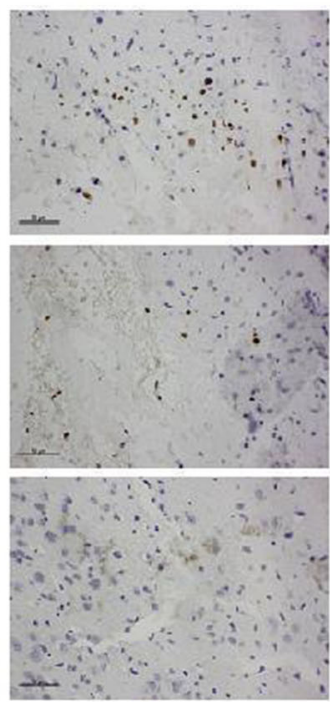

72

Time following trauma (h)

C57

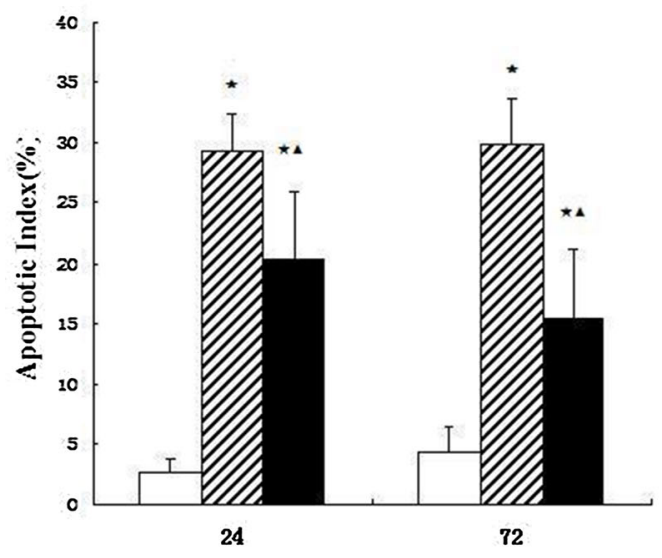

Nrf-/-

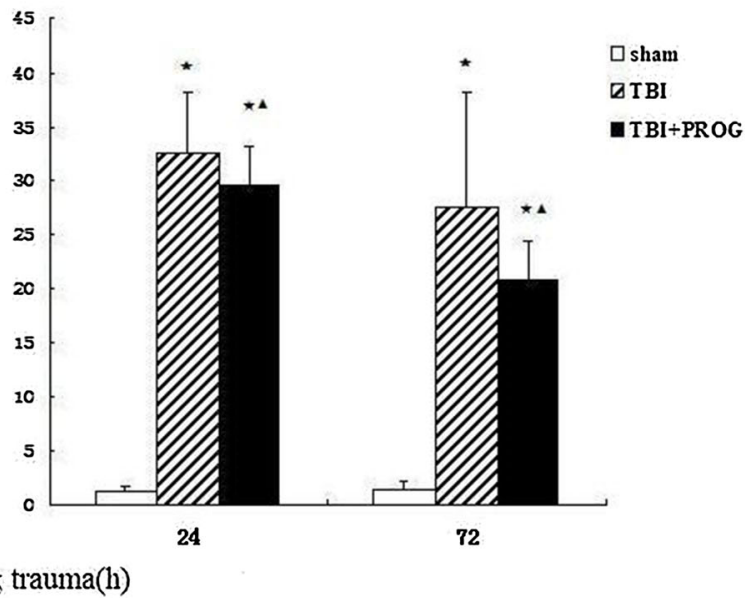

Fig. 2 TUNEL staining of $(\times 400)$ of apoptotic neurons in the cortex surrounding the injured site in sham group, TBI group, and TBI + PROG group $(n=12)$ between the C57 mice and Nrf2-/mouse model of brain injury at 24 and $72 \mathrm{~h}$ after injury. The right-

pleiotropic protective effects of PROG on TBI, consistent with a specific role for Nrf2/ARE signaling pathway. Collectively, we speculated that the pleiotropic neuroprotective effect of PROG on TBI is related to the Nrf2/ARE signal pathway. The results from our study confirm and extend these previous observations and demonstrate for the first time that post-injury administration of PROG may activate the Nrf2/ARE signal pathway, and this may be the mechanism underlying the pleiotropic neuroprotective effect of PROG on TBI. hand side illustrates the Nrf2-/- mouse model of TBI. The left-hand side is the C57 mice model of TBI. Scale bars, $50 \mu \mathrm{m}$. The apoptotic index is given as the mean \pm SE. $* P<0.01$ versus sham control and $\boldsymbol{\Delta}_{P}<0.01$ versus TBI vehicle-treated injured mice

Recently, two large, multicenter, randomized, doubleblind, placebo-controlled phase III clinical trials evaluated the effectiveness of PROG in patients with TBI. The results from both trials revealed that application of PROG would not be beneficial in the treatment of TBI [29, 30]. Various factors have been proposed which may have contributed to the failure of TBI trials to demonstrate convincing efficacy in the TBI population. One major issue is the heterogeneous nature of TBI. Another reason may be that the neuroprotective mechanisms of PROG on TBI are still 

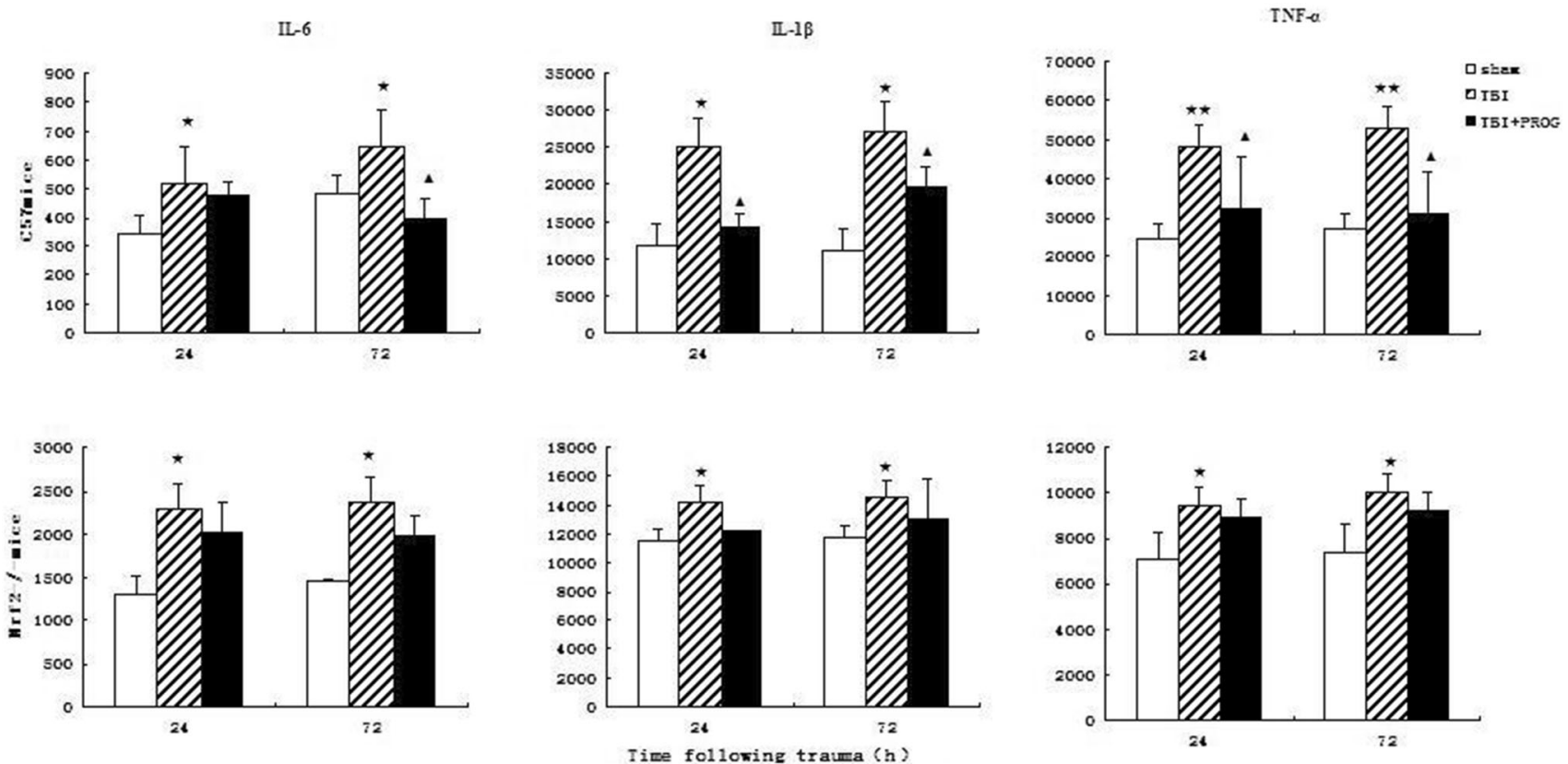

Fig. 3 Changes of inflammatory mediators in sham group, TBI group, and TBI + PROG group $(n=12)$ between the C57 mice and Nrf2-l- mouse model of brain injury at 24 and $72 \mathrm{~h}$ after injury. The

unclear. After extensive research on the treatment of PROG in TBI over the last three decades, it is clear that PROG is a neurosteroid that affects multiple mechanisms involved in neuroprotection and neural repair following various types of brain injury. These effects were associated with a reduction in edema [31] and reduce neurogenic inflammation [32]. PROG can upregulate the anti-apoptotic version of Bcl-2 and anti-apoptotic protein ERK [33, 34]. There is evidence that PROG can reduce lipid peroxidation [15] and attenuate neuronal excitotoxicity [13, 35, 36]. PROG can promote both central and peripheral remyelination through increasing myelin production [37-39]. In the current study, we found that PROG administration following TBI reduces apoptotic cell death and edema, and inhibits neurogenic inflammation and neuronal excitotoxicity in the brain tissue surrounding the cortical contusion, which was also observed by previous investigators.

PROG possesses pleiotropic neuroprotective effects that may markedly attenuate the injury cascade associated with TBI. However, the mechanism whereby PROG provides the effects on TBI is entirely unclear. Recently, the Nrf2/ ARE signal pathway has been shown to play an indispensable role in attenuation multiple pathophysiological processes in brain injury, including oxidative stress, mitochondrial dysfunction, and inflammation. TBI can induce Nrf2/ARE pathway activation in the brain [40]. Furthermore, activation of the Nrf2/ARE pathway by a pharmacologic inducer is able to protect neurons in the animal models of Parkinson's disease, cerebral ischemia, cytokine levels are given as the mean \pm SE. $* P<0.05$ and ${ }^{* *} P<0.01$ versus sham control and ${ }^{\boldsymbol{\Delta}} P<0.05$ versus TBI vehicle-treated injured mice

and intracerebral hemorrhage [41-43]. Specifically, the promising Nrf2/ARE activator sulforaphane has been demonstrated to protect neurons from TBI, including blood-brain-barrier dysfunction, brain edema formation, and cognitive deficits [44]. Another small molecule, carnosic acid, was shown to be neuroprotective in vivo in an animal model of cerebral ischemia. These protective effects of carnosic acid were also shown to be mediated by activation of the Nrf2/ARE pathway and hence through downstream mediators that are capable of eliciting a robust neuroprotective effect [45].

However, none of the previous studies have focused on the Nrf2/ARE signaling pathway in relation to the pleiotropic neuroprotection of PROG after TBI, despite the demonstrated role of PROG in neuroprotection. To further verify the causal relation between the Nrf2/ARE pathway activation by PROG and its pleiotropic neuroprotective effect in TBI, we used the TBI model of Nrf2-knockout mice. In the current study, we found that the pleiotropic neuroprotective effect of PROG on TBI reduced cerebral edema, apoptosis, inflammatory reaction, and intracellular calcium ion overload effects after TBI in Nrf2 $+/+$ mice but not in mice lacking the Nrf2 gene, confirming both the neuroprotective action and the Nrf2 dependence of PROG in vivo. This study revealed that the Nrf2/ARE signaling pathway could be activated when treated with PROG. The findings reported here suggest for the first time that the Nrf2/ARE signal pathway may be involved in the pleiotropic neuroprotective effect of PROG on TBI. 

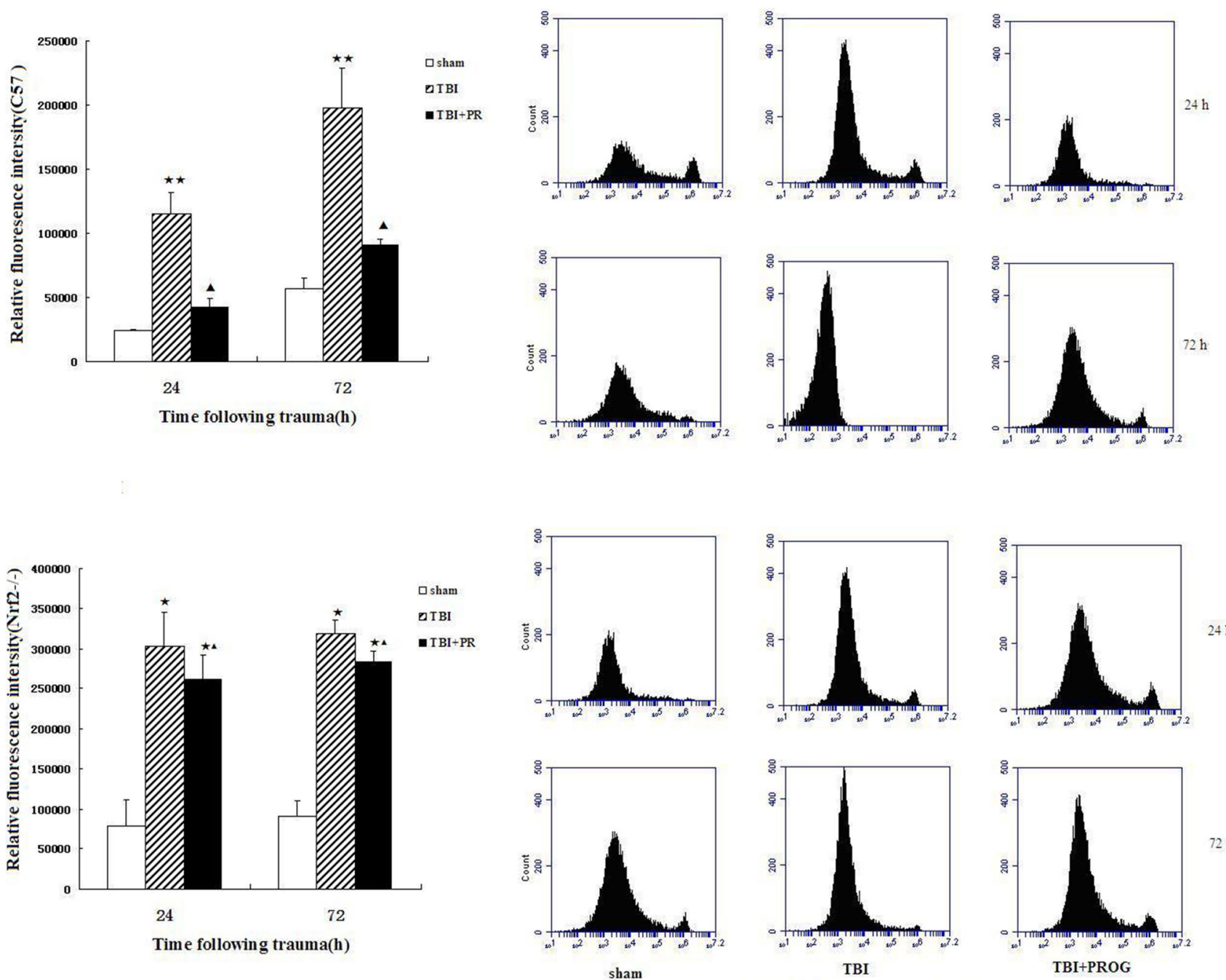

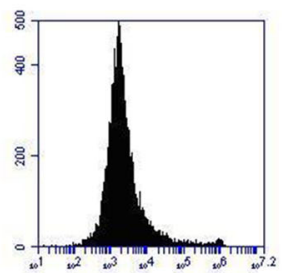

TBI

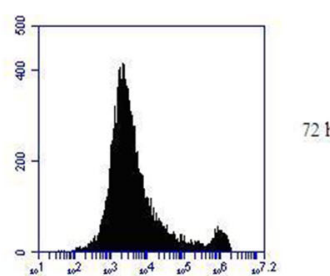

TBI+PROG

Relative fluorescence intensity

Fig. 4 Relative fluorescence intensity of intracellular free calcium in sham group, TBI group, and TBI + PROG group $(n=12)$ in the C57 mice and Nrf2-/- mouse model of brain injury at 24 and $72 \mathrm{~h}$ after trauma. The right-hand side illustrates the flow cytometric analysis of brains in sham group, TBI group, and TBI + PROG group of C57 mice and Nrf2-/ - mice brain tissues at 24 and $72 \mathrm{~h}$ after injury. The

In summary, to the best of our knowledge, this is the first study to demonstrate an effect of PROG on the Nrf2/ARE signaling pathway in the injured brain after TBI. The benefit of PROG administration after TBI may be due to its salutary effect on modulating the Nrf2/ARE signaling pathway. However, the study only presented preliminary evidence that PROG could exert pleiotropic neuroprotective effect against TBI via up-regulating the Nrf2/ARE signaling pathway. The present experimental results do not fully support this conclusion. Further experiments will be required to determine whether PROG exerts its pleiotropic neuroprotective effects via activation of Nrf2/ARE signaling pathways. This hypothesis could be tested by determining the upstream left-hand side is the quantitative presentation of the relative fluorescence intensity of intracellular free calcium. The upper line is the C57 mouse model of TBI. The lower line is the Nrf2-I- mouse model of TBI. The relative fluorescence intensity of intracellular free calcium is given as the mean \pm SE. $* P<0.05$ and ${ }^{*} P P<0.01$ versus sham control and ${ }^{\mathbf{\Lambda}} P<0.05$ versus TBI vehicle-treated injured mice

regulators of Nrf2/ARE, promoter binding sites, and the downstream effector genes of PROG.

Acknowledgments This work was supported by Grants from Zhejiang provincial natural science foundation; Hangzhou science and technology development plans; Zhejiang provincial and Hangzhou medical and health science and technology project; and Zhejiang key laboratory of organ development and regeneration, China.

Authors' Contributions MZ and GX designed and conducted the study, analyzed data, prepared the figures, and wrote the manuscript; MZ, JW, and HD participated in study design and preparation of the manuscript. MZ, WW, GX, and HD performed TUNEL labeling and ELISA analysis. JW and GX performed flow cytometric analysis and evaluated cerebral edema. 


\section{Compliance with Ethical Standards}

Conflict of interest There are no conflicts of interest.

Open Access This article is distributed under the terms of the Creative Commons Attribution 4.0 International License (http://creativeco mmons.org/licenses/by/4.0/), which permits unrestricted use, distribution, and reproduction in any medium, provided you give appropriate credit to the original author(s) and the source, provide a link to the Creative Commons license, and indicate if changes were made.

\section{References}

1. Andriessen TM, Horn J, Franschman G, Van der Naalt J, Haitsma I, et al. Epidemiology, severity classification, and outcome of moderate and severe traumatic brain injury: a prospective multicenter study. J Neurotrauma. 2011;28:2019-31.

2. McIntosh TK, Smith DH, Meaney DF, Kotapka MJ, Gennarelli TA, et al. Neuropathological sequelae of traumatic brain injury: relationship to neurochemical and biomechanical mechanisms. Lab Invest. 1996;74:315-42.

3. Yi JH, Hazell AS. Excitotoxic mechanisms and the role of astrocytic glutamate transporters in traumatic brain injury. Neurochem Int. 2006;48:394-403.

4. Faden AI, Demediuk P, Panter SS, Vink R. The role of excitatory amino acids and NMDA receptors in traumatic brain injury. Science. 1989;244:798-800.

5. Tyurin VA, Tyurina YY, Borisenko GG, Sokolova TV, Ritov $\mathrm{VB}$, et al. Oxidative stress following traumatic brain injury in rats: quantitation of biomarkers and detection of free radical intermediates. J Neurochem. 2000;75:2178-89.

6. Lewen A, Matz P, Chan PH. Free radical pathways in CNS injury. J Neurotrauma. 2000;17:871-90.

7. Frugier T, Morganti-Kossmann MC, O'Reilly D, McLean C. In situ detection of inflammatory mediators in post mortem human brain tissue after traumatic injury. J Neurotrauma. 2010;27:497-507.

8. Ghirnikar RS, Lee YL, Eng LF. Inflammation in traumatic brain injury: role of cytokines and chemokines. Neurochem Res. 1998;23:329-40.

9. Raghupathi R. Cell death mechanisms following traumatic brain injury. Brain Pathol. 2004;14:215-22.

10. Donkin JJ, Vink R. Mechanisms of cerebral edema in traumatic brain injury: therapeutic developments. Curr Opin Neurol. 2010;23:293-9.

11. Stein DG. A clinical/translational perspective: can a developmental hormone play a role in the treatment of traumatic brain injury? Horm Behav. 2013;63:291-300.

12. Wei J, Xiao GM. The neuroprotective effects of progesterone on traumatic brain injury: current status and future prospects. Acta Pharmacol Sin. 2013;34:1485-90.

13. Smith SS. Progesterone administration attenuates excitatory amino acid responses of cerebellar Purkinje cells. Neuroscience. 1991;42:309-20.

14. Djebaili M, Guo Q, Pettus EH, Hoffman SW, Stein DG. The neurosteroids progesterone and allopregnanolone reduce cell death, gliosis, and functional deficits after traumatic brain injury in rats. J Neurotrauma. 2005;22:106-18.

15. Roof RL, Hoffman SW, Stein DG. Progesterone protects against lipid peroxidation following traumatic brain injury in rats. Mol Chem Neuropathol. 1997;31:1-11.

16. Pan DS, Liu WG, Yang XF, Cao F. Inhibitory effect of progesterone on inflammatory factors after experimental traumatic brain injury. Biomed Environ Sci. 2007;20:432-8.
17. Baulieu EE, Robel P. Neurosteroids: a new brain function? J Steroid Biochem Mol Biol. 1990;37:395-403.

18. Schumacher M, Robel P, Baulieu EE. Development and regeneration of the nervous system: a role for neurosteroids. Dev Neurosci. 1996;18:6-21.

19. Kato J, Hirata S, Nozawa A, Yamada-Mouri N. Gene expression of progesterone receptor isoforms in the rat brain. Horm Behav. 1994;28:454-63.

20. Collado ML, Rodriguez-Manzo G, Cruz ML. Effect of progesterone upon adenylate cyclase activity and cAMP levels on brain areas. Pharmacol Biochem Behav. 1985;23:501-4.

21. Singh M. Ovarian hormones elicit phosphorylation of Akt and extracellular signal regulated kinase in explants of the cerebral cortex. Endocrine. 2001;14:407-15.

22. Migliaccio A, Piccolo D, Castoria G, Di Domenico M, Bilancio A, et al. Activation of the Src/p21ras/Erk pathway by progesterone receptor via cross-talk with estrogen receptor. EMBO J. 1998;17:2008-18.

23. Chen G, Shi J, Jin W, Wang L, Xie W, et al. Progesterone administration modulates TLRs/NF-kappaB signaling pathway in rat brain after cortical contusion. Ann Clin Lab Sci. 2008;38:65-74.

24. Owuor ED, Kong AN. Antioxidants and oxidants regulated signal transduction pathways. Biochem Pharmacol. 2002;64:765-70.

25. Hong Y, Yan W, Chen S, Sun CR, Zhang JM. The role of Nrf2 signaling in the regulation of antioxidants and detoxifying enzymes after traumatic brain injury in rats and mice. Acta Pharmacol Sin. 2010;31:1421-30.

26. Jaiswal AK. Nrf2 signaling in coordinated activation of antioxidant gene expression. Free Radic Biol Med. 2004;36:1199-207.

27. Itoh K, Chiba T, Takahashi S, Ishii T, Igarashi K, et al. An Nrf2/ small Maf heterodimer mediates the induction of phase II detoxifying enzyme genes through antioxidant response elements. Biochem Biophysical Res Commun. 1997;236:313-22.

28. Feeney DM, Boyeson MG, Linn RT, Murray HM, Dail WG. Responses to cortical injury: i. Methodology and local effects of contusions in the rat. Brain Res. 1981;211:67-77.

29. Skolnick BE, Maas AI, Narayan RK, van der Hoop RG, MacAllister T, Ward JD. A clinical trial of progesterone for severe traumatic brain injury. New Engl J Med. 2014;371:2467-76.

30. Wright DW, Yeatts SD, Silbergleit R, Palesch YY, Hertzberg VS, Frankel M. Very early administration of progesterone for acute traumatic brain injury. New Engl J Med. 2014;371:2457-66.

31. Guo Q, Sayeed I, Baronne LM, Hoffman SW, Guennoun R, Stein DG. Progesterone administration modulates AQP4 expression and edema after traumatic brain injury in male rats. Exp Neurol. 2006;198:469-78

32. Limmroth V, Lee WS, Moskowitz MA. GABAA-receptor-mediated effects of progesterone, its ring-A-reduced metabolites and synthetic neuroactive steroids on neurogenic edema in the rat meninges. Br J Pharmacol. 1996;117:99-104.

33. Djebaili M, Hoffman SW, Stein DG. Allopregnanolone and progesterone decrease cell death and cognitive deficits after a contusion of the rat pre-frontal cortex. Neuroscience. 2004;123:349-59.

34. Stein DG, Wright DW. Progesterone in the clinical treatment of acute traumatic brain injury. Expert Opin Investig Drugs. 2010;19:847-57.

35. Luoma JI, Kelley BG, Mermelstein PG. Progesterone inhibition of voltage-gated calcium channels is a potential neuroprotective mechanism against excitotoxicity. Steroids. 2011;76:845-55.

36. Shen H, Gong QH, Yuan M, Smith SS. Short-term steroid treatment increases delta GABAA receptor subunit expression in rat CA1 hippocampus: pharmacological and behavioral effects. Neuropharmacology. 2005;49:573-86. 
37. De Nicola AF, Gonzalez SL, Labombarda F, Deniselle MC, Garay L, et al. Progesterone treatment of spinal cord injury: effects on receptors, neurotrophins, and myelination. J Mol Neurosci. 2006;28:3-15.

38. Gonzalez SL, Labombarda F, Gonzalez Deniselle MC, Guennoun R, Schumacher M, De Nicola AF. Progesterone up-regulates neuronal brain-derived neurotrophic factor expression in the injured spinal cord. Neuroscience. 2004;125:605-14.

39. Labombarda F, Gonzalez S, Gonzalez Deniselle MC, Garay L, Guennoun R, et al. Progesterone increases the expression of myelin basic protein and the number of cells showing NG2 immunostaining in the lesioned spinal cord. J Neurotrauma. 2006;23:181-92.

40. Yan W, Wang HD, Hu ZG, Wang QF, Yin HX. Activation of Nrf2-ARE pathway in brain after traumatic brain injury. Neurosci Lett. 2008;431:150-4.

41. Chen PC, Vargas MR, Pani AK, Smeyne RJ, Johnson DA, et al. Nrf2-mediated neuroprotection in the MPTP mouse model of
Parkinson's disease: critical role for the astrocyte. Proc Natl Acad Sci USA. 2009;106:2933-8.

42. Shih AY, Li P, Murphy TH. A small-molecule-inducible Nrf2mediated antioxidant response provides effective prophylaxis against cerebral ischemia in vivo. J Neurosci. 2005;25:10321-35.

43. Zhao X, Sun G, Zhang J, Strong R, Dash PK, et al. Transcription factor Nrf2 protects the brain from damage produced by intracerebral hemorrhage. Stroke. 2007;38:3280-6.

44. Zhao J, Kobori N, Aronowski J, Dash PK. Sulforaphane reduces infarct volume following focal cerebral ischemia in rodents. Neurosci Lett. 2006;393:108-12.

45. Satoh T, Kosaka K, Itoh K, Kobayashi A, Yamamoto M, et al. Carnosic acid, a catechol-type electrophilic compound, protects neurons both in vitro and in vivo through activation of the Keap1/ Nrf2 pathway via S-alkylation of targeted cysteines on Keap1. J Neurochem. 2008;104:1116-31. 